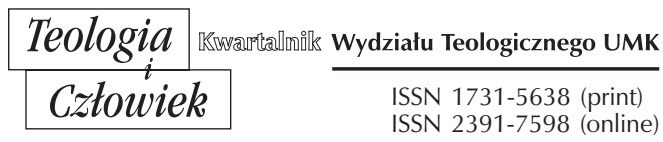

(ब) $(1)$

49(2020)1, ss. 27-42

\title{
ALCUNE LINEE DI ONTOLOGIA TRINITARIA
}

DOI: http://dx.doi.org/10.12775/TiCz.2020.002

Riassunto. Larticolo presenta la storia di un concetto chiamato ontologia trinitaria. Lautore descrive come emerge questa idea nel pensiero di stampo cristiano, partendo dalle ituizioni di Sant'Agostino. Avendo analizzato il concetto di persona riferito tanto a Dio quanto all'uomo, si è presentato un testo di un teologo tedesco, Klaus Hemmerle, da cui ha preso le mossa la ricerca contemporanea in proposito. Di seguito, si è analizzato i testi scelti di Piero Coda, in cui lui si occupa di unontologia trinitaria.

Parole chiavi: ontologia trinitartia; Sant’Agostino; Klaus Hemmerle; Piero Coda.

Abstract. Lines of a Trinitarian Ontology. The article presents the history of a concept called Trinitarian ontology. The author describes how this idea emerges in Christian-style thinking, starting from the ituitions of Saint Augustine. Having analyzed the concept of person referred to both God and man, a text was presented by a German theologian, Klaus Hemmerle, from which the contemporary research on the subject was based. Below, the selected texts by Piero Coda were analyzed, in which he deals with a Trinitarian ontology.

Key words: trinitarian ontology; Saint Augustin; Klaus Hemmerle; Piero Coda.

Streszczenie. Niektóre linie ontologii trynitarnej. Artykuł przedstawia zarys historii koncepcji nazywanej ontologią trynitarną. Autor opisuje, jak ta idea wyłania się w myśli chrześcijańskiej, poczynając od św. Augustyna. Po przeanalizowaniu pojęcia 
osoby odnoszącej się zarówno do Boga, jak i do człowieka, omawia się tekst pewnego niemieckiego teologa, Klausa Hemmerlego, od publikacji którego rozpoczęły się współczesne badania $\mathrm{w}$ omawianej sprawie. W publikacji przeanalizowano ponadto wybrane teksty Piero Cody, w których ten włoski myśliciel zajmuje się ontologią trynitarną.

Słowa kluczowe: ontologia trynitarza; św. Augustyn; Klaus Hemmerle; Piero Coda.

\section{INTRODUZIONE}

Ontologia trinitaria deriva dalla teologia trinitaria e, partendo dalla rivelazione neotestamentaria, sviluppa, in modi diversi nelle diverse epoche, una linea di pensiero ontologico. ${ }^{1}$ Se per ontologia trinitaria intendiamo il contemplare il riflesso del mistero della Trinità nel creato, allora possiamo affermare che essa, in un certo senso, è sempre stata.

E opportuno affrontare questo discorso e quindi questo articolo vuole offrire ciò per presentare un riassunto del contemporaneo pensiero italiano alla communità scientifica polacca affinché essa ne prenda le mosse per contribuire alla ricerca di quel genere.

Il testo presenterà in un primo momento alcune nozioni storiche, evidenziando come un concetto di unontologia trinitaria si faceva presente nel pensiero cristiano. Poi, si discuterà il contenuto di un libro di $\mathrm{K}$. Hemmerle intitolato Tesi di unontologia trinitaria in quanto esso sia, in un certo senso, il punto di partenza dell'idea odierna del concetto analizzato. Infine, saranno evidenziati i fili conduttori provenienti dall'Italia soprattutto dalla scuola costruitasi attorno alle intuizioni di Chiara Lubich.

\section{STORIA E FONTI DEL CONCETTO}

In Agostino d'Ippona si trova teorizzata la presenza analogica della Trinità nell'uomo, ${ }^{2}$ particolarmente nella sua componente spirituale: ${ }^{3}$ in

${ }^{1}$ Cfr. E. Iezzoni, Ontologia trinitaria: dal mistero della Rivelazione una sfida per la filosofia contemporanea, "Nuova Umanità" 170 (2007) 2, 187.

${ }^{2}$ Cfr. L.F. Ladaria, Il Dio vivo e vero. Il mistero della Trinità, Ed. San Paolo, Milano 2012, 299-300.

${ }^{3}$ Cfr. Gn 1, 26. 
essa, infatti, l'essere, il conoscere e il volere sono intimamente connessi, pur nella loro diversità, e richiamano rispettivamente il Padre, il Figlio e lo Spirito. ${ }^{4}$ Largomento è approfondito successivamente ne La Trinità,${ }^{5}$ in cui il santo aveva rivisitato in modo piuttosto originale le categorie del pensiero greco, avendo avviato un cambiamento sostanziale in campo trinitario, di cui oggi si gode i frutti: ancorandosi alla Scrittura, infatti, Sant'Agostino poteva pensare l'essere di Dio come Amore, senza per questo abbandonare il rigore del procedere razionale.

La verità fondamentale, per lui, è che "Dio è senza dubbio sostanza o, se il termine è più proprio, essenza, che i greci chiamano ousia [...] dal verbo esse si è fatto derivare essentia". ${ }^{6}$ Quindi, dopo aver dimostrato la perfetta identità delle persone divine a livello di essenza ${ }^{7}$ e di perfezioni assolute, ${ }^{8}$ Agostino concludeva che la distinzione fra loro deve essere cercata altrove e cioè nella categoria della relazione, dove quest'ultima non ha lo stesso contenuto dell'accidente aristotelico, ma si riferisce all'essere di Dio in modo sostanziale: si afferma l'unità dellessenza e la distinzione dei Tre. ${ }^{9}$ In altri termini Agostino ha osservato che se si parla in Dio di Padre, Figlio e Spirito, ciò vuol dire che in lui vi sono delle relazioni - paternità, figliazione e donazione ${ }^{10}$ - e che queste relazioni non significano tanto

${ }^{4}$ Cfr. Sant'Agostino d'Ippona, Confessionum, XIII, 11, 12 [trad. it. Le confessioni, NBA, Città Nuova, Roma 1965].

${ }^{5}$ Cfr. Sant'Agostino d'Ippona, De Trinitate, X, 11, 17 - X, 12, 19 [trad. it. La Trinità, NBA, Città Nuova, Roma 1973]. D'ora in poi cit. come La Trinità. Cfr. anche P. Coda, Il De Trinitate di Agostino e la sua promessa, "Nuova Umanità" 140-141 (2002) 3-4, 219-248.

${ }^{6}$ La Trinità, V, 2, 3.

7 Per approfondire argomento veda A. Clemenzia, In unum con-venire. L'unità ecclesiale in Agostino di Ippona, Città Nuova, Roma 2015, 123-135.

${ }^{8}$ Cfr. La Trinità, VII, 1, 2.

9 Cfr. La Trinità, V, 5, 6. Cfr. anche L.F. Ladaria, La Trinità mistero di comunione, Ed. Paoline, Milano 2004, 95-96; P. Coda, Dalla Trinità. L'avvento di Dio tra storia e profezia, Città Nuova, Roma 2011, 378.

${ }^{10}$ Lo Spirito Santo è chiamato nella Scrittura "dono di Dio" (cfr. Gv 4, 10; At 8, 20). Da questa permessa Sant'Agostino trae che anche nei riguardi allo Spirito possiamo parlare usando il concetto della relazione. Cfr. La Trinità, V, 11, 12. Cfr. anche P. Milano, Persona in teologia. Alle origini del significato di persona nel cristianesimo antico, Ed. Dehoniane, Napoli 1984, 301-302. 
diversità, quanto distinzione. ${ }^{11}$ In Dio, tutto è sostanziale, pertanto queste relazioni non possono essere accidenti: dal momento che sfuggono alla temporalità e perciò alla mutazione, sono eterne. ${ }^{12} \mathrm{Ne} \mathrm{La} \mathrm{Città} \mathrm{di} \mathrm{Dio,} \mathrm{il}$ santo d'Ippona afferma ancora una volta che "il Padre ha certamente il Figlio ma non egli è il Figlio, il Figlio ha il Padre ma non egli è il Padre. Dunque in base agli attributi che si dicono in senso assoluto e non relativo, in Dio si identificano essere e avere". ${ }^{13}$

Il teologo d'Ippona, introducendo la categoria della relazione ad invicem in un contesto trinitario, presenta quindi lo Spirito Santo come intratrinitario Amor Patris et Filii, l'Amore tra l'Amante e l'Amato: Amor et dilectio, che permetterà di contemplare le relazioni trinitarie nella reciprocità, come amore dato e ricambiato. ${ }^{14}$

${ }^{11}$ La Trinità, V, 5, 6. Ivi: "si parla a volte di Dio secondo la relazione; così il Padre dice relazione al Figlio e il Figlio al Padre, e questa relazione non è accidente, perché l'uno è sempre Padre, l'altro sempre Figlio" anche se "non è la stessa cosa esser Padre ed essere Figlio, tuttavia la sostanza non è diversa, perché questi appellativi non appartengono all'ordine della sostanza, ma della relazione; relazione che non è un accidente, perché è mutevole". I due dati, uno dalla filosofia e pure dalla rivelazione veterotestamentaria, l'altro proveniente da quella nuovo testamentaria, sono qui accolti in modo tale che l'unità di colui che è non è infranta dall'alterità di Padre, di Figlio e di Spirito la quale non dice altro che la non accidentale relazione dell'Uno all'Altro che sempre sono coloro che sono.; P. Sguazzardo, Sant'Agostino e la teologia trinitaria del XX secolo. Ricerca storico-ermeneutica e prospettive speculative, Città Nuova, Roma 2006, 512; P. Sguazzardo, Unità e trinità in Dio secondo Agostino d'Ippona, "Path" 11 (2012) 2, 327-346.

${ }^{12}$ Cfr. La Trinità, V, 4, 5. "La sostanza dice esse ad se, mentre la relazione dice esse ad aliud. La relazione non può dunque ridursi alla sostanza. In Dio si distinguono delle perfezioni assolute che si riferiscono a se stesse e delle perfezioni che si riferiscono ad alium: le prime sono comuni, le seconde stabiliscono le distinzioni fra i Tre; quelle si predicano substantialiter; queste relative. Il Padre allora non si distingue dalla divinità, santità e così via, ma solo dal Figlio". Milano, op. cit., 302.

${ }_{13}$ Sant'Agostino d'Ippona, De civitate Dei, XI, 10, 1 [trad. it. Città di Dio, NBA, Città Nuova, Roma 1988]. Sottolineatura mia. Questo principio sarà riformulato dal Concilio di Firenze (1442), che dogmatizzerà che in Dio omniaque sunt unum, ubi non obviat relationis oppositio. Bolla Cantate Domino sull'unione con i copti e gli etiopi (il 4 febbraio 1442), in Denz 1330.

${ }^{14}$ Cfr. La Trinità, VIII, 10, 14. Per un approfondimento veda: Clemenzia, op. cit., 158-165. 
Malgrado i Padri della Chiesa si sforzassero di spiegare l'evento di Cristo alla luce dell'unità divina, si avvertiva l'insufficienza dell'ontologia da loro adoperata, ${ }^{15}$ e questo vale anche per Agostino, a proposito della riflessione sul concetto di persona, ${ }^{16}$ a cui non si può non dedicare almeno un accenno in questo articolo perché unontologia trinitaria vuole spiegare la realtà a partire dall'unitrinità di $\mathrm{Dio}^{17}$ e questo è sempre riferito alla nozione della persona. ${ }^{18}$

Il termine ipostasi o persona vuole precisare che il Figlio e lo Spirito Santo non sono né qualcosa di increato né degli esseri intermedi, della cui presenza ci si trova nel giudaismo vetero- e intertestamentario, ${ }^{19}$ né attributi di Dio o modi di rivelazione dell'unico Dio.

Nel IV secolo, i cosiddetti Padri Cappadoci sono i primi a riflettere sulle ipostasi di Dio in modo sistematico e, con il concetto di ipostasi, tutti e tre vogliono sottolineare ciò che differenzia una realtà da un'altra. ${ }^{20}$ D’altro canto, con la sua formula tres personae - una substantia ${ }^{21}$ è Tertulliano il primo teologo ad utilizzare il termine persona nell'ambito

${ }^{15}$ Cfr. P. Coda, L'altro di Dio. Rivelazione e kenosi in Siergiej Bulgakov, Città Nuova, Roma 1998, 87-89.

${ }^{16}$ Per un accenno sull'etimologia del lemma veda M.J. Farrelly, The Trinity. Rediscovering the Central Christian Mytery, Rowman\&Littlefield Publisher 2005, 219-224.

${ }^{17}$ Cfr. A. Clemenzia, Ontología y razón trinitaria, "Isidorianum" 22 (2013) 44, 384. Cfr. anche Id., op. cit., 71-72.

18 "Il concetto e la specifica comprensione di persona trovano la propria origine nella teologia cristiana, in particolare nel suo sforzo di denominazione dal punto di vista del linguaggio e di cogliere concettualmente due misteri centrali della fede: in primo luogo la realtà di Gesù Cristo, così come risulta costituita dall'interagire e dal mutuo rapportarsi dell'essere-uomo ed essere-Dio [...] e in secondo luogo - soprattutto - la realtà della rivelazione cristiana, che si mostra nella 'tensione' tra essere-uno ed esseretre, tra unità e diversità". G. Greshake, Il Dio Unitrino. Teologia trinitaria, Queriniana, Brescia 2000, 79.

19 Per approfondire veda: P. Gamberini, Un Dio relazione. Breve manuale di dottrina trinitaria, Città Nuova, Roma 2007, 27-33. Cfr. anche Ladaria, Il Dio..., op. cit., 263-280; B. Sesboüé, Il mistero della Trinità: Riflessione speculativa ed elaborazione del linguaggio. Il "Filioque". Le relazioni trinitarie, in: B. Sesboüé, J. Wolinski (edd.), Storia dei dogmi, vol. 1: Il Dio della salvezza, Piemme, Casale Monferrato (AL) 1996, 263-268.

${ }^{20}$ Gamberini, op. cit., 44.

${ }^{21}$ Tertulliano, Adversus Praxeam, PL 2, 180. 
della teologia trinitaria, distinguendo la persona, che indica la differenza - in quanto manifesta qualcosa di individuale, dotato di caratteristiche peculiari e per cui in Dio vi è distinto e caratteristico -, dalla sostanza, che denota ciò che è comune. ${ }^{22}$

Per quanto riguarda il magistero agostiniano, ${ }^{23}$ il termine persona costituisce "un nome generico, tanto che lo si può applicare anche all'uomo, sebbene sia così grande la distanza tra l'uomo e Dio", ${ }^{24}$ e il suo impiego nel discorso sulla Trinità è indispensabile, in quanto ci permette di distinguere il piano dell'essenza divina ed il piano della sua sussistenza (hypóstasis) relazionale. ${ }^{25}$ In altre parole, Sant’Agostino, nell'elaborare la dottrina trinitaria, pone in stretto rapporto il termine "persona" e quello di "relazione", in quanto il primo indica appunto la relazione del Padre, del Figlio e dello Spirito. ${ }^{26}$

Lautore di De civitate Dei, tuttavia, non offre una vera e propria definizione della persona, che, invece, darà Severino Boezio, come naturae rationalis individua substantia. ${ }^{27} \mathrm{Il}$ discorso boeziano prende avvio da una preoccupazione teologica: confutare le eresie cristologiche, avanzate da Eutiche e Nestorio. Quest'ultimo negava l'unità del Cristo, affermando che vi sarebbero due persone in lui. Boezio precisa che in Cristo vi è una duplice natura, una divina e una umana, ma non una duplice persona, ponendo allo stesso tempo le basi della futura riflessione antropologico-personalista di stampo cristiano: la persona è tale in quanto dotata di una natura razionale, ma soprattutto in quanto sostanza individuale. ${ }^{28}$

${ }^{22}$ Cfr. Gamberini, op. cit., 44-45.

${ }^{23}$ Nonostante le sue obiezioni verso il termine "persona", il santo d'Ippona lo adopera perché nell'epoca della composizione di De Trinitate esso era già entrato nel linguaggio ufficiale della Chiesa all'interno della professione di fede del I Concilio di Costantinopoli.

${ }^{24}$ La Trinità, V, 6.

${ }^{25}$ Cfr. Farrelly, op. cit., 225.

${ }^{26}$ Cfr. Gamberini, op. cit., 45; Ladaria, Il Dio..., op. cit., 309-314. Per un approfondimento veda Clemenzia, op. cit., 136-158.

${ }^{27}$ Boethius, Liber de persona et duabus naturis contra Eutychem et Nestorium, III (PL 64, 1343).

${ }^{28}$ Cfr. E. Simonotti, La persona: individualità e relazione. Itinerari dell'umanesimo cristiano, "Sapienza" 58 (2005) 4, 401. 
Alla ricerca trinitaria agostiniana, si rifarà San Tommaso d'Aquino, che trova in questo modo buone premesse per condurre la teologia sul versante ontologico e penetrare il mistero trinitario con un pensiero fortemente metafisico. L'Aquinate spiega che la paternità, la figliazione e la spirazione passiva sono tre relazioni sussistenti ${ }^{29}$ e quindi sono le tre "persone" o ipostasi divine. ${ }^{30}$ Le tre persone divine hanno anche nomi propri: $^{31}$ la prima si chiama Padre, in quanto genera il Figlio; ingenito, perché non deriva da nessuno; principio, perché da lui derivano il Figlio e lo Spirito Santo. ${ }^{32}$ La seconda persona si chiama Figlio, in quanto viene generata dal Padre; verbo, perché è termine del pensiero divino; immagine, perché riproduce sostanzialmente il Padre.$^{33}$ La terza, invece, si chiama Amore, in quanto termine della volizione; Dono, perché in essa Dio si dà; Spirito, perché va spirata dal Padre e dal Figlio. ${ }^{34}$

Per Tommaso, il termine "persona", riferito alla Trinità, indica relazione, ${ }^{35}$ mentre, attribuito all'essere umano, significa individualità. ${ }^{36}$

Date queste permesse generali, possiamo approfondire l'indagine, anche perché, come sottolinea A. Clemenzia, in realtà il contributo degli autori citati, e di tanti altri, va ben oltre il desiderio iniziale del

29 Per un approfondimento veda Ladaria, Il Dio..., op. cit., 322-327; Sesboüé, op. cit., 282-287.

${ }^{30}$ Cfr. STh I q. 29, a. 4. Qui il Dottor Angelico fa presente che "persona" indica individualità, quando si predica dell'essere umano ("la persona in generale, come si è detto, significa una sostanza individua di natura ragionevole"), mentre quando se la riferisce all'essere del Dio-Trinità essa indica relazione: "la persona divina significa una relazione come sussistente".

31 "Il nome proprio di una persona significa ciò che la distingue da tutte le altre". STh I, q. 33, a. 2.

32 STh I, q. 33.

33 STh I, qq. 34-35.

${ }^{34}$ STh I, qq. 37-38. Qui il Dottor Angelico afferma che l'amore come tale è una caratteristica che riguarda Dio nella sua essenza e perciò tutte e tre le persone divine. Distinguendo fra amore essenziale che si riferisce alle persone ed alla loro unità, ed amore personale o nozionale, quest'ultimo lo attribuisce allo Spirito Santo.

${ }^{35}$ Cfr. STh I, q. 14, a. 2, ad 1.

${ }^{36}$ Per approfondimento veda G. Bertuzzi, Natura e persona in San Tommaso, a proposito della personalità ontologica di Cristo, "Divus Thomas" 115 (2012) 1, 90-118. 
semplice contemplare il riflesso del mistero del Dio Uno e Trino nella creazione. ${ }^{37}$

\section{TESI DI ONTOLOGIA TRINITARIA DI K. HEMMERLE E IL SUO IMPATTO ALLE RICERCA CONTEMPORANEA}

Si può partire da uno scritto di K. Hemmerle, intitolato Tesi di ontologia trinitaria, ${ }^{38}$ dove "il tema dell'ontologia trinitaria viene riproposto in forma programmatica". 39

Hemmerle afferma che la teologia ha bisogno di un'ontologia, mostrando come, in sua assenza, si riduca in qualcosa di altro: nella storia delle religioni, ${ }^{40}$ nell'antropologia, ${ }^{41}$ nelletica, ${ }^{42}$ nella testimonianza di fede. ${ }^{43}$ D’altro canto, non solo la teologia necessita di un'ontologia, ma anche la filosofia, che, pur potendo limitarsi alla filosofia della scienza, alla logica, alletica della vita personale o sociale, deve giustificare i motivi di questa scelta, che, appunto, "pervengono però inevitabilmente allambito dell'ontologia", ${ }^{44}$ poiché anche "chi sostiene che la domanda sullessere non ha senso, ha già compiuto una scelta preliminare sul senso dell'essere". ${ }^{45}$

Affermando l'inevitabilità del ritorno dei due saperi all'ontologia, K. Hemmerle invita filosofi e teologi ad elaborare, in una relazione di interscambio - giustificata storicamente, perché, come nota l'autore delle Tesi, "nel corso della storia spesso, anzi fondamentalmente sempre, la teologia si è rifatta, per formulare il proprio specifico, a scelte preliminari

37 Clemenzia, art. cit., 385, nota 9.

${ }^{38} \mathrm{~K}$. Hemmerle, Tesi di ontologia trinitaria. Per un rinnovamento del pensiero cristiano, Città Nuova, Roma 1996.

${ }^{39}$ L. Žák, Permessa: Verso una ontologia trinitaria, in L. Žák, P. Coda (edd.), Abitando la Trinità. Per un rinnovamento dell'ontologia, Città Nuova, Roma 1998, 11.

${ }^{40}$ Hemmerle, op. cit., 26-27.

${ }^{41}$ Ibidem, 27.

${ }^{42}$ Ibidem

${ }^{43}$ Ibidem, 27-28.

${ }^{44}$ A. Frick, Le Tesi di ontologia trinitaria di K. Hemmerle. Un nuovo inizio, in P. Coda, A. Tapken (edd.), La Trinità e il pensare. Figure percorsi prospettive, Città Nuova, Roma 1997, 286.

${ }^{45}$ Hemmerle, Tesi, 28. 
e precomprensioni ontologiche proprie del pensiero coevo oppure ricevute in eredità"; ${ }^{46}$ d'altronde, anche la teologia ha spinto gli studiosi ad assegnare a questi concetti filosofici un'importanza di gran lunga maggiore, rispetto al passato, ${ }^{47}$ favorendo la nascita di una "nuova ontologia", fondata sullo specifico della fede in Gesù Cristo: l'"evento trinitario". ${ }^{48}$ Occorre dunque domandarsi: "in che modo si trasformano dal di dentro le esperienza e le concezioni fondamentali che l'uomo ha di Dio, del mondo e dell'uomo, quando vi irrompe la fede in Gesù Cristo?". ${ }^{49}$

Dal momento che Dio ha donato il suo Figlio, obbediente fino alla morte, il nostro essere è chiamato ad una conversione, che consiste nel "credere all'amore", spostando il baricentro da sé verso l'A/altro. ${ }^{50}$ L'agápe compresa come dono di sé, come essere-per l'altro - è il "ritmo" dell'essere di Dio, che si rivela all'uomo in Gesù Cristo, che dona se stesso sulla croce; un "ritmo", al quale occorre aderire, per ritrovare le origini e il senso del proprio essere. ${ }^{51}$ Qui appaiono i concetti fondamentali per costruire, anzi per riscoprire una nuova ontologia: l'agápe e il darsi, i cui approcci sono quelli dall'accadere, dal compiersi. ${ }^{52}$ Un'ontologia trinitaria propone di attribuire un nome al Dio Uno-Trino, quello di amore, come ciò che si dona e si comunica, nella relazione che non obbliga, ma, al contrario, lascia libero ogni ente di rispondere, accogliendo o meno il dono. ${ }^{53}$

Il teologo tedesco nota che la novità di quella ontologia consiste nel fatto che essa prende le mosse dal mistero di Dio Trino ${ }^{54}$ e che quel mistero altro non è che l'amore; anzi, l'amore che si dona, tramite Gesù Cristo, ${ }^{55}$ che da una parte rivela il Padre, mentre, dall'altra, è da lui glorificato. ${ }^{56}$

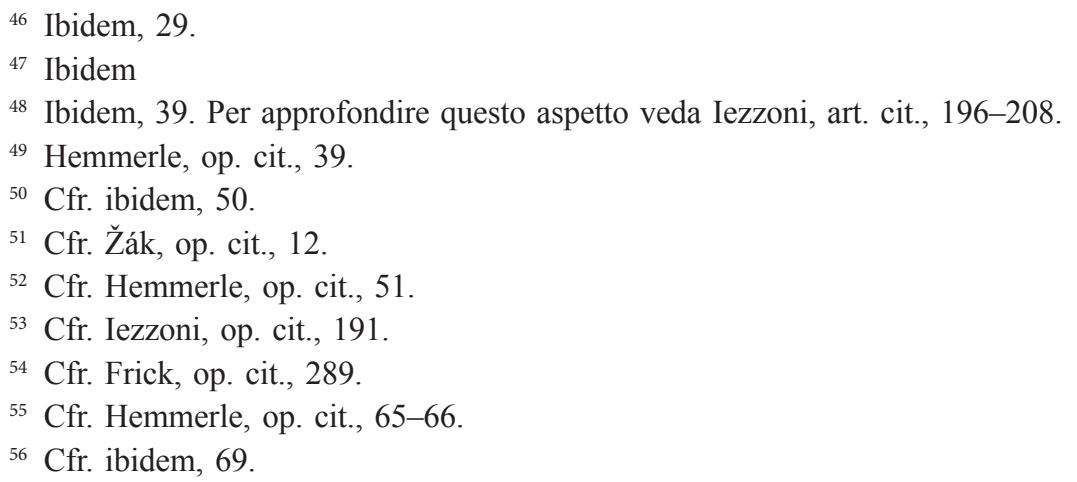


"Ma fondare l'ontologia in chiave trinitaria rappresentava, per Hemmerle, unesigenza legata non solo al rinnovamento della filosofia e della teologia, ma anche della società. Egli, infatti, era del tutto persuaso che, se è vero che oggi, come forse mai in precedenza, la fallacia del pensiero, la caducità dell'essere, la problematicità del soggetto, l'assenza di Dio, i pericoli che minacciano la libertà e il senso sono diventati pane quotidiano, è altrettanto vero che lontologia trinitaria - se recepita come invito a una nuova prassi di vita - potrebbe sollecitare a una 'nuova società fondata sul «modello trinitario»". 57

Detto questo, per prima cosa si può affermare che unontologia trinitaria sarà quel novum, attraverso il quale si vuole cogliere il significato dell'ente. Essa parte dall'analisi dell'amore reciproco delle persone divine, rivelato nell'evento di Gesù Cristo, visto come dono.

Detto ciò si va ad accennare allo sviluppo che questo concetto ha ricevuto in Italia, dove unontologia trinitaria è stata portata avanti, in modo particolare, da Piero Coda. ${ }^{58}$

Tuttavia, a questo proposito, non si può non partire da Bruno Forte, che sin dall'inizio della sua attività teologica mostra di essere profondamente convinto dell'importanza di pensare l'universale e i particolari della verità rivelata "a partire dallo specifico cristiano della fede nel Dio trinitario."59 Del resto, lui riconosce nel mistero pasquale "il centro delleconomia della salvezza, il luogo sempre vivo della dispensazione dell'amore trinitario per gli uomini", perché è nella prospettiva pasquale-trinitaria che diviene possibile "tracciare le linee di un'ontologia trinitaria, intesa come la riflessione sullessere degli enti sviluppata a partire dall'accadere originario e sempre nuovo, che è l'evento della donazione creatrice operata dalla Trinità e rivelata pienamente nella kenosi del Venerdì Santo e nella gloria di Pasqua: la Trinità si offre come il mistero del mondo, la sua profondità ultima e originaria, la sua origine e il suo grembo trascendenti, che segnano di sé tutto ciò che esiste"..$^{60}$

57 Žák, op. cit., 12.

${ }^{58}$ Una prova di condurre il discorso entro orizzonte di un'ontologia trinitaria è stata adoperata da M.F. Sciacca, nel suo Ontologia triadica e trinitaria. Discorso metafisico teologico, Marzorati, Milano 1976.

59 B. Forte, Trinità come storia, Ed. Paoline, Milano 1988, 14.

${ }^{60}$ B. Forte, Teologia della storia. Saggio sulla rivelazione, l'inizio e il compi- 
Parlando di ontologia trinitaria, non si può non riferirsi a Piero Coda, in quanto autore di Evento Pasquale. Trinità e storia. Elaborando la sua proposta di ontologia trinitaria, lui parte dalle intuizioni espresse nelle Tesi di Hemmerle, dimostrando, allo stesso tempo, una grande sensibilità per le idee e prospettive ontologico-trinitarie del pensiero di stampo protestante e ortodosso. Nel suo primo saggio: Evento Pasquale con il significativo sottotitolo Genesi, significato e interpretazione di una prospettiva emergente nella teologia contemporanea. Verso un progetto di ontologia trinitaria, lo studioso mette in evidenza l'emergente tendenza, in alcuni teologi, di affrontare il discorso in una prospettiva pasquale-trinitaria. ${ }^{61}$

Secondo Coda, è inevitabile incamminarsi verso un "progetto" di ontologia trinitaria (ontologia del mistero dell'essere come dischiuso dall'evento pasquale), grazie al quale sarebbe finalmente possibile articolare i tre fondamentali livelli del mistero cristiano - creazione, evento pasquale, ${ }^{62}$ mistero trascendente della Trinità - e prevedere, di conseguenza, un'analogia entis all'interno dell'analogia fide ${ }^{63}{ }^{63}$ ovvero un'analogia creationis, ${ }^{64}$ un'analogia crucis $^{65}$ e un'analogia amoris ovvero analogia Trinitatis ${ }^{66}$

L'intenzione del teologo italiano è innanzitutto quella di mettere in luce ciò che nelle Tesi di Hemmerle è stato annunciato, ma non molto ap-

mento, Ed. Paoline, Milano 1991, 267.

${ }^{61}$ Cfr. P. Coda, Gesù Crocifisso e abbandonato e la Trinità. III. Analogia del Cristo e dello Spirito, "Nuova Umanità" 28-29 (1983), 31-80.

${ }^{62}$ Cfr. P. Coda, Gesù Crocifisso e abbandonato e la Trinità. IV. Analogia Trinitatis, "Nuova Umanità" 32 (1984), 60.

${ }^{63}$ Cfr. ibidem, 79.

64 "L'essere creato personale è posto in essere come imago del Padre nel Verbo per poi essere assunto dal Verbo stesso come sua propria espressione nel mistero dell'Incarnazione". Ibidem

65 'L'essere personale creato è condotto nella libertà dal Verbo incarnato a 'perdersi' sulla Croce nella sua autonomia creaturale assolutisticamente intesa, ed anche a 'convertire' la dinamica della sua stessa libertà che nel peccato si pone contro Dio e qui si collocherebbe la giusta esigenza di una dialettica negativa centrata sulla Croce, come momento interiore dell'affermazione analogica". Ibidem

66 "L'essere personale creato, che si è perso con e in Cristo crocifisso e abbandonato, 'si ritrova' con Lui e in Lui per lo Spirito Santo nella koinonía trinitaria della risurrezione e della trasfigurazione ecclesiale - e questo è il livello dove possono risultare preziosi gli sviluppi della teologia orientale". Ibidem 
profondito dal punto di vista teologico: l'assoluta centralità e inevitabilità della realtà di Cristo crocifisso e abbandonato, perché in e da lui viene assunta e redenta ogni realtà dell'essere creaturale, persino la negazione esistenziale della vocazione della creaturalità (trascendersi in Dio), compiuta dalla libera decisione dell'uomo stesso. ${ }^{67}$

Cristo, sulla croce e nellabbandono, sperimenta come uomo-Dio le conseguenze di questo rifiuto, "ma ne fa come Dio-uomo l'analogia reale e strumentale del dono-di-Sé al Padre, e del dono agli uomini della relazione che lo unisce e lo distingue al e dal Padre: lo Spirito Santo". "In tal modo - continua a spiegare P. Coda -, Cristo crocifisso e risorto non solo ri-dona all'uomo la relazione col Padre, ma gli dona il compimento della vocazione di questa relazione: la vita nello Spirito (pericoresi teandrica come partecipazione alla pericoresi trinitaria). In essa, l'interpersonalità ecclesiale diventa, per grazia, analogia reale della Trinità, in seno alla Trinità, e segno e strumento (sacramento) nella storia della partecipazione universale e cosmica a questa vocazione escatologica, fino a che «Dio sarà tutto in tutti»" 68

Coda ritorna a parlare di ontologia trinitaria in vari altri interventi, sottolineando la centralità del concetto del "non-essere" e affermando che il pensiero metafisico classico lo adopera in due direzioni: assoluta, quando di una cosa si dice che essa non è, e relativa, che dichiara che una cosa non è l'altra. ${ }^{69}$ Esiste, però, un altro modo per impiegare questo

${ }^{67}$ Cfr. P. Coda, Il Cristo Crocifisso e Abbandonato redenzione della libertà e nuova creazione, "Nuova Umanità" 105-106 (1996) 3-4, 365-400. Cfr. anche P. Coda, Evento Pasquale. Trinità e Storia. Genesi, significato e interpretazione di una prospettiva emergente nella teologia contemporanea. Verso un progetto di ontologia trinitaria, Città Nuova, Roma 1984, 161.

${ }^{68}$ Coda, Evento Pasquale, 177.

${ }^{69}$ P. Coda, Postilla sulla semantica del "non-essere" in teologia, in: P. Coda, J. Tremblay, A. Clemenzia (edd.), Il nulla-tutto dell'Amore. La teologia come sapienza del Crocifisso, Città Nuova, Roma 2013, 201. È possibile parlare anche di non-essere come "negazione, a livello esistenziale, della relazione ontologica di creaturalità che lega la persona a Dio nella libertà (= negazione «negativa»): questa negazione ha come frutto un non-essere perché nega ciò che è l'essere della persona creata (la costitutiva relazionalità a Dio). Ma, abbiamo notato, questa negazione è possibile perché la libertà è in radice la possibilità che la persona è di negar-si (negazione «positiva») come immanenza autosufficiente, per trascendersi in Dio - attuando così il suo essere come atto 
concetto. Nota, infatti, Coda che, "per affermare logicamente e ontologicamente, nel rigore del discorso teologico, la reale distinzione/alterità dei Tre nell'unica essenza divina", sant'Agostino dice che "l'uno non è l'altro, essendo però ciascuno l'unico vero Dio". ${ }^{70}$ Questa nozione del non-essere è chiamata il "non-essere relativo" e fa sì che le persone divine, allo stesso tempo, siano-Uno e siano-distinte. In questo senso, il 'non-essere' appare come "atto più profondo dell'essere delle divine Persone". "Ogni divina Persona, proprio perché non è, è: perché non è sussistenza chiusa in sé, ma è sussistenza che è dono senza residui di sé (e dunque, in qualche maniera, rinunciando a sé, "non è"), proprio per questo è se stessa, è Persona divina nell'unità-distinzione con altre divine Persone, nella unità-unicità dell'Essere divino come Amore." ${ }^{71}$ Di conseguenza, il "non-essere" nell'amore di Cristo - che introduce nel mistero del rapporto d'unità-nella-distinzione delle persone divine - permette di trovare la via d'accesso, per poter pensare il mistero della creazione e della divinizzazione delle persone create, ovvero il rapporto di unità e distinzione tra il Creatore e la creatura e, successivamente, anche il rapporto intersoggettivo.

Come Hemmerle, così anche Coda analizza attentamente il concetto dell'agápe, perché "la rivelazione cristiana dischiude l'orizzonte dell'essere come Agápe, e cioè come libero, gratuito, reciproco ed effusivo dono di sé. È questo il novum della rivelazione ebraica, che giunge a inaspettato compimento in Cristo. Lo dicono l'incarnazione del Figlio di Dio, la sua morte in croce e risurrezione, l'essere di Dio come Trinità, la creazione, la salvezza, ecc."72

Secondo Coda, quindi, la filosofia e la teologia contemporanee si trovano di fronte all'esigenza di elaborare unontologia che sarà fondata sulla rivelazione cristiana e, quindi, che sarà capace di esprimere il più

di relazione a Lui. La negazione esistenziale della creaturalità è dunque, per così dire, negazione (negativa) della negazione (positiva)". P. Coda, Gesù Crocifisso e abbandonato e la Trinità. II. Creazione Croce Trinità: Una permessa sull'analogia, "Nuova Umanità" 24-25 (1982-1983), 45, nota 33.

${ }^{70} \mathrm{P}$. Coda, L'unità e la Trinità di Dio nel ritmo di un'ontologia trinitaria, "Sophia: Ricerche su i fondamenti e la correlazione dei saperi” 2 (2010) 2, 178.

${ }^{71}$ P. Coda, Il negativo e la Trinità. Ipotesi su Hegel, Città Nuova, Roma 1987, 402.

${ }^{72}$ Coda, Dalla Trinità..., op. cit., 559. 
adeguatamente possibile l'ermeneutica pasquale e trinitaria del mistero cristiano. ${ }^{73}$ In questo senso, la categoria del "non-essere" (dono di sé) introiettata nella comprensione dell'essere come condizione di possibilità della sua immanente struttura trinitaria - appare come il primo passo sulla via di una tale elaborazione.

Lasciando dunque nuovamente la parola a P. Coda, si può affermare che "il lemma «ontologia trinitaria» può essere preso in due sensi: uno più largo e uno più stretto. In senso largo, ontologia trinitaria designa ogni interpretazione della realtà che - esplicitamente o anche implicitamente - muova dal luogo entro cui l'evento di Gesù Cristo ci ha attirati. [...] In questo senso - e ciò è senźaltro un tema da approfondire - unontologia trinitaria può costruire l'orizzonte interpretativo di riferimento, libero e plurale, di ogni interpretazione particolare della realtà messo in opera dai diversi saperi. [...] Cè poi un significato più stretto ed epistemologicamente preciso di 'ontologia trinitaria', secondo cui questo lemma nomina quella specifica interpretazione dell'essere in quanto essere scaturisce dalla presa di coscienza formalmente istituita del luogo in cui Gesù ci ha attirati tenendo conto della sua rilevanza propriamente ontologica". ${ }^{74}$

Volendo riassumere questi brevi pensieri, dobbiamo quindi dire che unontologia trinitaria vuole cogliere lo specifico della realtà alla luce dell'essere divino che si rivela in Gesù Cristo come l'unione delle tre persone e perciò come l'amore. ${ }^{75}$

${ }^{73}$ Cfr. P. Coda, Il negativo..., op. cit., 414.

${ }^{74}$ P. Coda, L'ontologia trinitaria: che cos'è?, "Sophia: Ricerche su i fondamenti e la correlazione dei saperi” 4(2012)2, 165. Cfr. anche P. Coda, Trinità: 2. L'ontologia trinitaria, in: J.-Y. Lacoste (ed.), Dizionario critico di Teologia, Borla-Città Nuova, Roma 2005, 1412-1415; L. Žák, Unità di Dio: quaestio princeps dell'ontologia trinitaria, "Path" 11(2012)2, 442.

${ }^{75}$ Dio in Gesù di Nazaret si è fatto uomo ed in questo atto si è rivelato un Dio in tre persone e questa auto-rivelazione comporta la redenzione del mondo. Come giustamente sottolinea Clemenzia: 'l' 'ontologia' considera l'essere, anzi, il dinamismo che caratterizza tutto ciò che 'è', al di là di una sua possibile funzionalità. 'Trinitaria', invece, proprio perché non funge da sostantivo, ma da aggettivo, è in qualche modo la qualificazione di ciò di cui si sta parlando (nel nostro caso l'ontologia). 'Trinitaria', in particolare, allude alla prospettiva da cui si osserva, e dunque al luogo da cui si guarda una particolare realtà". Clemenzia, op. cit., 69-70. 


\section{BIBLIOGRAFIA}

Bertuzzi G., Natura e persona in San Tommaso, a proposito della personalità ontologica di Cristo, "Divus Thomas" 115 (2012) 1, 90-118.

Boethius, Liber de persona et duabus naturis contra Eutychem et Nestorium, III (PL 64, 1343).

Clemenzia A., In unum con-venire. L'unità ecclesiale in Agostino di Ippona, Città Nuova, Roma 2015.

Clemenzia A., Ontología y razón trinitaria, "Isidorianum” 22 (2013) 44, 381-399.

Coda P., Dalla Trinità. L’avvento di Dio tra storia e profezia, Città Nuova, Roma 2011.

Coda P., Evento Pasquale. Trinità e Storia. Genesi, significato e interpretazione di una prospettiva emergente nella teologia contemporanea. Verso un progetto di ontologia trinitaria, Città Nuova, Roma 1984.

Coda P., Gesù Crocifisso e abbandonato e la Trinità. II. Creazione Croce Trinità: Una permessa sullanalogia, "Nuova Umanità" 24-25 (1982-1983), 25-68.

Coda P., Gesù Crocifisso e abbandonato e la Trinità. III. Analogia del Cristo e dello Spirito, "Nuova Umanità" 28-29 (1983), 31-80.

Coda P., Gesù Crocifisso e abbandonato e la Trinità. IV. Analogia Trinitatis, "Nuova Umanità” 32(1984), 53-80.

Coda P., Il Cristo Crocifisso e Abbandonato redenzione della libertà e nuova creazione, "Nuova Umanità" 105-106 (1996)3-4, 365-400.

Coda P., Il De Trinitate di Agostino e la sua promessa, "Nuova Umanità" 140-141 (2002) 3-4, 219-248.

Coda P., Il negativo e la Trinità. Ipotesi su Hegel, Città Nuova, Roma 1987.

Coda P., L'altro di Dio. Rivelazione e kenosi in Siergiej Bulgakov, Città Nuova, Roma 1998.

Coda P., Lontologia trinitaria: che cosèe?, "Sophia: Ricerche su i fondamenti e la correlazione dei saperi" 4 (2012) 2, 159-170.

Coda P., Lunità e la Trinità di Dio nel ritmo di unontologia trinitaria, "Sophia: Ricerche su i fondamenti e la correlazione dei saperi" 2 (2010) 2, 174-189.

Coda P., Postilla sulla semantica del "non-essere" in teologia, in Coda P., Tremblay J., Clemenzia A. (edd.), Il nulla-tutto dell'Amore. La teologia come sapienza del Crocifisso, Città Nuova, Roma 2013, 200-209.

Coda P., Trinità: 2. Lontologia trinitaria, in: J.-Y. Lacoste (ed.), Dizionario critico di Teologia, Borla-Città Nuova, Roma 2005, 1412-1415.

Farrelly M.J., The Trinity. Rediscovering the Central Christian Mytery, Rowman\&Littlefield Publisher 2005.

Forte B., Teologia della storia. Saggio sulla rivelazione, l'inizio e il compimento, Ed. Paoline, Milano 1991.

Forte B., Trinità come storia, Ed. Paoline, Milano 1988.

Frick A., Le Tesi di ontologia trinitaria di K. Hemmerle. Un nuovo inizio, in Coda P., Tapken A. (edd.), La Trinità e il pensare. Figure percorsi prospettive, Città Nuova, Roma 1997, 331-371. 
Gamberini P., Un Dio relazione. Breve manuale di dottrina trinitaria, Città Nuova, Roma 2007.

Greshake G., Il Dio Unitrino. Teologia trinitaria, Queriniana, Brescia 2000.

Hemmerle K., Tesi di ontologia trinitaria. Per un rinnovamento del pensiero cristiano, Città Nuova, Roma 1996.

Iezzoni E., Ontologia trinitaria: dal mistero della Rivelazione una sida per la filosofia contemporanea, "Nuova Umanità"170 (2007) 2, 187-224.

Ladaria L.F., Il Dio vivo e vero. Il mistero della Trinità, Ed. San Paolo, Milano 2012.

Ladaria L.F., La Trinità mistero di comunione, Ed. Paoline, Milano 2004.

Milano P., Persona in teologia. Alle origini del significato di persona nel cristianesimo antico, Ed. Dehoniane, Napoli 1984.

Sant'Agostino d'Ippona, Confessionum [trad. it. Le confessioni, NBA, Città Nuova, Roma 1965].

Sant'Agostino d'Ippona, De civitate Dei, XI, 10, 1 [trad. it. Città di Dio, NBA, Città Nuova, Roma 1988].

Sant’Agostino, De Trinitate [trad. it. La Trinità, NBA, Città Nuova, Roma 1973].

Sciacca M.F., Ontologia triadica e trinitaria. Discorso metafisico teologico, Marzorati, Milano 1976.

Sesboüé B., Il mistero della Trinità: Riflessione speculativa ed elaborazione del linguaggio. Il "Filioque". Le relazioni trinitarie, in: Sesboüé B., Wolinski J. (edd.), Storia dei dogmi, vol. 1: Il Dio della salvezza, Piemme, Casale Monferrato (AL) 1996, 263-268.

Sguazzardo P., Sant'Agostino e la teologia trinitaria del XX secolo. Ricerca storico-ermeneutica e prospettive speculative, Città Nuova, Roma 2006.

Sguazzardo P., Unità e trinità in Dio secondo Agostino d'Ippona, "Path" 11 (2012) 2, 327-346.

Simonotti E., La persona: individualità e relazione. Itinerari dell'umanesimo cristiano, “Sapienza" 58 (2005) 4, 401-418.

STh I qq. 14; 29; 33-35; 37-38.

Tertulliano, Adversus Praxeam, PL 2, 180.

Žák L., Permessa: Verso una ontologia trinitaria, in Žák L., Coda P. (edd.), Abitando la Trinità. Per un rinnovamento dellontologia, Città Nuova, Roma 1998, 5-25.

Žák L., Unità di Dio: quaestio princeps dellontologia trinitaria, “Path" 11 (2012) 2, 439-464. 